\title{
Suplementação com ferro na criança saudável até aos dois anos de idade
}

\section{Revisão Baseada na Evidência}

Luís Filipe Cavadas, ${ }^{*}$ Ana Nunes, ${ }^{* *}$ Rosa Branca Santos, ${ }^{* * *}$ Joana Melo Adrião****

\section{RESUMO}

Objectivo: Determinar se a suplementação de rotina com ferro, na criança saudável até aos dois anos de idade, influencia o desenvolvimento psicomotor, antropometria e a morbimortalidade.

Fontes de dados: Base de dados MEDLINE e sítios de Medicina Baseada na Evidência.

Métodos de revisão: Pesquisa de revisões baseadas na evidência, normas de orientação clínica, meta-análises, revisões sistemáticas e ensaios clínicos controlados e aleatorizados, utilizando os termos MeSH: Iron, Infant, Dietary Supplements, de artigos publicados desde Janeiro de 2000 até Dezembro de 2009, em Inglês, Francês, Espanhol e Português. Para avaliar a qualidade dos estudos e a força de recomendação, foi utilizada a escala Strength of Recommendation Taxonomy da American Family Physician.

Resultados: Foram identificados 163 estudos, dos quais foram seleccionados nove. Uma norma de orientação clínica, duas revisões baseadas na evidência, duas meta-análises, uma revisão sistemática e três ensaios clínicos cumpriram os critérios de inclusão. Não existe influência no desenvolvimento psicomotor, antropometria e morbimortalidade, nas crianças saudáveis sem factores de risco suplementadas com ferro. Nas crianças com risco aumentado para anemia ferropénica a suplementação revela-se benéfica.

Conclusões: Não existe evidência de benefício da suplementação com ferro por rotina nas crianças sem factores de risco até aos dois anos de idade. É aconselhada suplementação com ferro por rotina até aos doze meses de idade nas crianças que têm um risco aumentado para anemia ferropénica (Strength of Recommendation B - SOR B). São necessários mais estudos, controlados, aleatorizados, de elevada qualidade, sobre a suplementação com ferro, orientados para a criança saudável.

Palavras-chave: Ferro; Criança; Suplementos Dietéticos.

\section{INTRODUÇÃO}

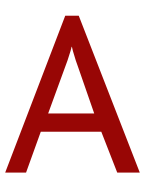
deficiência de ferro constitui o défice nutricional mais comum no mundo, sendo a principal causa de anemia na criança, sobretudo nos países em desenvolvimento. ${ }^{1-5}$

$\mathrm{O}$ aumento das necessidades de ferro devido a um rápido crescimento da massa muscular e volume de

\footnotetext{
* Interno de Medicina Geral e Familiar, Unidade de Saúde Familiar Lagoa, ACES de Matosinhos

** Interno de Medicina Geral e Familiar, Unidade de Saúde Familiar Oceanos, ACES de Matosinhos

*** Assistente de Medicina Geral e Familiar, Unidade de Saúde Familiar Lagoa, ACES de Matosinhos

**** Assistente de Medicina Geral e Familiar, Unidade de Saúde Familiar de Lavra, ACES de Matosinhos
}

sangue e a uma diminuição da sua absorção, juntamente com uma dieta contendo alimentos pobres em ferro ou com baixa biodisponibilidade, está na base destas alterações. ${ }^{1,6}$

Os recém-nascidos de termo apresentam reservas de ferro suficientes até cerca dos seis meses de idade., ${ }^{7,9}$, Posteriormente ficam dependentes da presença deste nutriente na dieta, sendo que práticas de má alimentação colocam muitas crianças em risco. Os recém-nascidos pré-termo ou com baixo peso não possuem reservas séricas de ferro ajustadas às suas necessidades, nomeadamente a uma taxa de crescimento superior, estando mais vulneráveis à anemia ferropénica. ${ }^{10,11}$ 
O ferro é um oligoelemento importante para o desenvolvimento da criança, intervindo em múltiplas funções no organismo ${ }^{3}$. Sendo assim, a anemia ferropénica tem um potencial impacto no desenvolvimento psicomotor e cognitivo, no comportamento, no crescimento e na maturação dos órgãos sensoriais (visão e audição). ${ }^{1,2,5}$ Mas quando se trata de implementar a suplementação de rotina com ferro a todas as crianças é preciso ponderar os benefícios e os riscos. ${ }^{10}$

A administração de ferro deve ser cuidadosamente controlada, uma vez que este, em excesso, pode interferir com a absorção de outros nutrientes ou gerar radicais livres que prejudicam as funções celulares e suprimem a actividade enzimática, associando-se a lesão tecidular, nomeadamente hepática. ${ }^{10}$ Alguns estudos relacionam o excesso de ferro ao comprometimento de uma evolução estaturoponderal adequada e a um aumento do risco de doença infecciosa. ${ }^{10}$

A preocupação com as repercussões do défice de ferro na saúde e desenvolvimento das crianças tem levado à criação de diferentes recomendações para a suplementação deste oligoelemento consoante a idade das crianças e a prevalência de anemia na população.

Por tudo isto é fundamental conhecer a melhor evidência disponível sobre a influência da suplementação com ferro em crianças.

Neste contexto, foi efectuada uma revisão baseada na evidência, com o objectivo de determinar se a suplementação de rotina com ferro, na criança saudável até aos dois anos de idade, influencia o desenvolvimento psicomotor, a antropometria e a morbimortalidade.

\section{MÉTODOS}

Foi realizada uma pesquisa nas bases de dados $M E$ DLINE, Guidelines Finder, National Guideline Clearinghouse, Canadian Medical Association Infobase, The Cochrane Library, DARE, Bandolier, TRIP e InfoPoems, de revisões baseadas na evidência, normas de orientação clínica, meta-análises, revisões sistemáticas e ensaios clínicos controlados e aleatorizados, publicados desde 1 de Janeiro de 2000 até 1 de Dezembro de 2009, em Inglês, Francês, Espanhol e Português, utilizando os termos MeSH: Iron, Infant, Dietary Supplements.

No Índex de Revistas Médicas Portuguesas, a pesquisa foi feita com a combinação dos termos da lista dos Descritores em Ciências da Saúde (DeCS), usando os descritores portugueses: Ferro, Lactente, Suplementos Dietéticos.

Os critérios utilizados para a inclusão dos artigos nesta revisão foram:

- População: crianças saudáveis, até aos dois anos de idade.

- Intervenção: suplementação de rotina com ferro.

- Comparação: suplementação com ferro versus não suplementação com ferro.

- Resultados: desenvolvimento psicomotor, antropometria e morbimortalidade.

Para avaliar a qualidade dos estudos e a força de recomendação, foi utilizada a escala de Strength of Recommendation Taxonomy (SORT) da American Family Physician (AFP)..$^{12}$ Segundo esta taxonomia, a qualidade do estudo está subdividida em três Níveis de Evidência (Nível de Evidência 1: estudos de boa qualidade, evidência orientada para o doente; Nível de Evidência 2: estudos de qualidade limitada, evidência orientada para o doente; e Nível de Evidência 3: outra evidência) e a Força de Recomendação em três graus (Força de Recomendação A: consistente, evidência orientada para o doente; Força de Recomendação B: inconsistente ou qualidade limitada, evidência orientada para o doente; e Força de Recomendação C: consensos, evidência orientada para a doença). ${ }^{12}$

\section{RESULTADOS}

A pesquisa efectuada resultou na identificação de 163 artigos, 12 dos quais realizados em Portugal. Os artigos repetidos foram excluídos.

Nove artigos foram seleccionados: uma norma de orientação clínica, ${ }^{1}$ duas revisões baseadas na evidência, ${ }^{2,6}$ duas meta-análises, ${ }^{13,14}$ uma revisão sistemática ${ }^{10}$ e três ensaios clínicos preencheram os critérios de in-

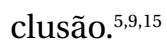

\section{Desenvolvimento psicomotor}

Segundo a norma de orientação clínica da United States Preventive Services Task Force (USPSTF) de 2006, a evidência é insuficiente para recomendar a suplementação de rotina com ferro nas crianças assintomáticas dos seis aos doze meses de idade. ${ }^{1}$ Segundo a mesma norma esta suplementação deve ser feita nas crianças assintomáticas dos seis aos doze meses de idade que têm um risco aumentado para anemia ferropénica. ${ }^{1}$ 
Na revisão baseada na evidência da American Family Physician de 2007, são referidas quais as crianças com risco aumentado para anemia ferropénica que devem ser suplementadas: pré-termo; baixo peso à nascença; crianças que vivem na pobreza; crianças que bebem leite de vaca como dieta principal; imigrantes de países em desenvolvimento; crianças de raça negra; nativos Americanos e do Alasca. ${ }^{2}$

Numa revisão baseada na evidência publicada também em 2007, é recomendado restringir a suplementação universal a populações com elevada prevalência de deficiência de ferro, taxas baixas de doenças infecciosas e bom acesso aos serviços saúde. ${ }^{6}$

A meta-análise de Sachdev HPS et al que analisou dezassete ensaios clínicos, incluindo 3646 crianças de países desenvolvidos e em desenvolvimento, concluiu não existir evidência consistente de que a suplementação com ferro tenha um efeito no desenvolvimento mental ou no desenvolvimento psicomotor da criança com menos de vinte e sete meses de idade. ${ }^{13}$

A revisão sistemática de Iannotti LL et al que incluiu vinte e seis ensaios clínicos, com um total de 55412 crianças de países em desenvolvimento, concluiu haver diminuição dos défices motores e cognitivos naquelas que apresentavam deficiência de ferro e que foram suplementadas. ${ }^{10}$

\section{Antropometria}

Os parâmetros antropométricos avaliados foram: comprimento, peso e perímetro cefálico.

A meta-análise de Ramakrishnan U et al analisou vinte e sete ensaios clínicos, com um total de 6671

\section{QUADRO I. Norma de Orientação Clínica e Revisões Baseadas na Evidência}

\begin{tabular}{|c|c|c|c|c|}
\hline & Referência & Resultados & Recomendações & $\begin{array}{l}\text { Força de } \\
\text { Recomendação }\end{array}$ \\
\hline NOC & $\begin{array}{l}\text { USPSTF } \\
(2006)^{1}\end{array}$ & $\begin{array}{l}\text { Benefício/Risco } \\
\text { Desenvolvimento } \\
\text { (fórmula enriquecida ou } \\
\text { suplementos) }\end{array}$ & $\begin{array}{l}\text { Evidência insuficiente para recomendar a } \\
\text { suplementação com ferro por rotina nas crianças } \\
\text { assintomáticas dos } 6 \text { aos } 12 \text { meses de idade. } \\
\text { Suplementação com ferro por rotina nas crianças } \\
\text { assintomáticas dos } 6 \text { aos } 12 \text { meses de idade que } \\
\text { têm um risco aumentado para Anemia ferropénica. }\end{array}$ & $\begin{array}{l}\text { I* } \\
\text { B* }\end{array}$ \\
\hline RBE & $\begin{array}{l}\text { Killip S et al. } \\
(2007)^{2}\end{array}$ & $\begin{array}{l}\text { Benefícios/Riscos } \\
\text { da suplementação de } \\
\text { ferro }\end{array}$ & $\begin{array}{l}\text { Suplementação com ferro por rotina nas crianças } \\
\text { assintomáticas dos } 6 \text { aos } 12 \text { meses de idade que } \\
\text { têm um risco aumentado para Anemia ferropénica: } \\
\text { Pré-termo, } \\
\text { Baixo peso à nascença, } \\
\text { Pobreza, } \\
\text { Leite de vaca como dieta principal, } \\
\text { Imigrantes de países em desenvolvimento, } \\
\text { Raça negra, nativos americanos e do Alasca. }\end{array}$ & B \\
\hline RBE & $\begin{array}{l}\text { Stoltzfus RJ } \\
\text { et al. }(2007)^{6}\end{array}$ & $\begin{array}{l}\text { Antropometria } \\
\text { Desenvolvimento } \\
\text { Morbilidade } \\
\text { Custo/Efectividade }\end{array}$ & $\begin{array}{l}\text { Restringir a suplementação universal a populações } \\
\text { com elevada prevalência de deficiência de ferro, } \\
\text { taxas baixas de doenças infecciosas e bom acesso } \\
\text { aos serviços de saúde. }\end{array}$ & B \\
\hline
\end{tabular}

Legenda: NOC - Norma de orientação clínica da United States Preventive Services Task Force (USPSTF), Força de Recomendação: I* - evidências são insuficientes para recomendar a favor ou contra a actividade preventiva, prova de que a actividade preventiva é insuficiente, de má qualidade, ou conflituosa; $\mathrm{B}^{*}$ - actividade preventiva recomendada, benefícios superam os riscos. RBE - Revisão Baseada na Evidência. Força de Recomendação B (Segundo taxonomia da American Family Physician) - inconsistente ou qualidade limitada, evidência orientada para o doente.

*Os autores optaram por apresentar nos resultados da NOC da USPSTF a taxonomia própria dessa entidade. 


\begin{tabular}{|c|c|c|c|c|c|}
\hline & Referência & População/Intervenção & Resultados & Conclusões & $\begin{array}{l}\text { Nível de } \\
\text { Evidência }\end{array}$ \\
\hline RS & $\begin{array}{l}\text { lannotti LL } \\
\text { et al. }(2006)^{10}\end{array}$ & $\begin{array}{l}26 \text { ECA } \\
\mathrm{n}=55412 \\
\text { Países em desenvolvimento } \\
\text { Crianças com } \\
<5 \text { anos de idade } \\
\text { Suplementação oral com ferro } \\
\text { Riscos/Benefícios }\end{array}$ & $\begin{array}{l}\text { Desenvolvimento } \\
\text { psicomotor } \\
\text { Antropometria } \\
\text { Morbilidade } \\
\text { Mortalidade }\end{array}$ & $\begin{array}{l}\text { Diminuição dos défices motores e } \\
\text { cognitivos nas crianças com } \\
\text { deficiência de ferro } \\
\text { Diminuição do ganho de peso nas } \\
\text { crianças com reservas de ferro } \\
\text { Maioria dos estudos sem efeito na } \\
\text { morbimortalidade }\end{array}$ & 2 \\
\hline MA & $\begin{array}{l}\text { Sachdev HPS } \\
\text { et al. }(2005)^{13}\end{array}$ & $\begin{array}{l}17 \text { ECA } \\
\mathrm{n}=3646 \\
\text { Países desenvolvidos e em } \\
\text { desenvolvimento } \\
\text { Crianças maioritariamente } \\
\text { saudáveis } \\
\text { Suplementação por via oral ou } \\
\text { parentérica }\end{array}$ & $\begin{array}{l}\text { Desenvolvimento } \\
\text { psicomotor }\end{array}$ & $\begin{array}{l}\text { Não existe evidência consistente } \\
\text { de que a suplementação com ferro } \\
\text { tenha um efeito no } \\
\text { desenvolvimento mental ou no } \\
\text { desenvolvimento motor da criança } \\
\text { com menos de } 27 \text { meses de idade. }\end{array}$ & 2 \\
\hline MA & $\begin{array}{l}\text { Ramakrishnan } \\
\text { U et al. } \\
(2009)^{14}\end{array}$ & $\begin{array}{l}27 \text { ECA } \\
\mathrm{n}=6671 \\
\text { Países desenvolvidos e em } \\
\text { desenvolvimento } \\
\text { Crianças saudáveis com menos } \\
\text { de } 5 \text { anos } \\
\text { Suplementação por via oral, } \\
\text { parentérica, fórmula ou cereais } \\
\text { enriquecidos }\end{array}$ & Antropometria & $\begin{array}{l}\text { Não existe um efeito positivo da } \\
\text { suplementação de ferro no peso e } \\
\text { altura das crianças. }\end{array}$ & 2 \\
\hline EC & $\begin{array}{l}\text { Yurdakok K } \\
\text { et al. }(2004)^{5}\end{array}$ & 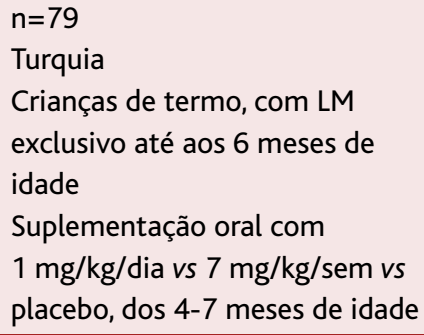 & Antropometria & $\begin{array}{l}\text { Sem influência no crescimento nas } \\
\text { crianças com reservas de ferro. }\end{array}$ & 2 \\
\hline EC & $\begin{array}{l}\text { Nagpal J et al. } \\
(2004)^{9}\end{array}$ & $\begin{array}{l}\mathrm{n}=100 \\
\text { Índia } \\
\text { Crianças de termo, entre os } 4 \text { e } \\
6 \text { meses de idade } \\
\text { Suplementação com ferro } \\
2 \text { mg/kg/dia vs placebo, } \\
\text { durante } 8 \text { semanas }\end{array}$ & Antropometria & Sem alterações na antropometria. & 2 \\
\hline EC & $\begin{array}{l}\text { Walker fischer } \\
\text { CL et al. } \\
(2009)^{15}\end{array}$ & $\begin{array}{l}\mathrm{n}=645 \\
\text { Bangladesh } \\
\text { Crianças entre os } 6 \text { e } 12 \text { meses } \\
\text { de idade } \\
\text { Suplementação com ferro } \\
20 \mathrm{mg} / 1 \text { vez por semana vs } \\
\text { placebo, durante } 6 \text { meses. }\end{array}$ & Antropometria & Sem alterações na antropometria. & 2 \\
\hline
\end{tabular}

Legenda: RS - revisão sistemática, MA - meta-análise, EC - ensaio clínico; $n$ - número amostral, ECA - estudo controlado e aleatorizado, LM - leite materno; Nível de Evidência 2 - estudo de qualidade limitada, evidência orientada para o doente. 


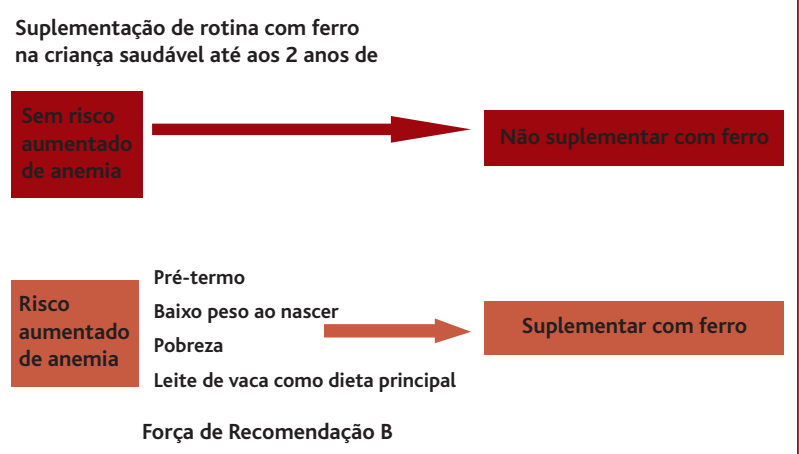

Figura 1. Recomendação para a suplementação de rotina com ferro na criança saudável até aos dois anos de idade.

crianças saudáveis menores de cinco anos provenientes de países desenvolvidos e em desenvolvimento. ${ }^{14}$ De acordo com os resultados analisados, concluiu não existir um efeito positivo da suplementação de ferro no peso e altura das crianças. A revisão sistemática de Iannotti LL et al verificou que naquelas crianças que apresentavam reservas de ferro e que foram suplementadas com este oligoelemento o ganho de peso foi adversamente afectado. ${ }^{10}$

Três ensaios clínicos realizados nos países em desenvolvimento, referiram não ser registada qualquer alteração nos parâmetros antropométricos nas crianças suplementadas com ferro. ${ }^{5,9,15}$

\section{Morbimortalidade}

Apenas foi avaliado este item na revisão sistemática de Iannotti LL et al, que conclui não haver alterações na morbilidade (nomeadamente aparecimento de doenças infecciosas, sinais e sintomas como febre e diarreia, bem como o número de hospitalizações) nem na mortalidade com a suplementação de ferro. ${ }^{10}$

São apresentados dois quadros que descrevem, respectivamente, as normas de orientação e revisões baseadas na evidência e as respectivas forças de recomendação (Quadro I) e as revisões sistemáticas, metaanálises e ensaios clínicos e os respectivos níveis de evidência (Quadro II).

\section{CONCLUSÕES}

Da análise de todos os estudos publicados até à data, não há evidência de benefício na suplementação de ferro por rotina nas crianças até aos dois anos e sem factores de risco (Força de Recomendação B).

É aconselhada a suplementação com ferro por rotina até aos doze meses de idade nas crianças que têm um risco aumentado para anemia ferropénica, que no caso de Portugal incluem principalmente os pré-termo ou com baixo peso à nascença (Força de Recomendação B).

De uma forma simplificada, é apresentado um esquema com as principais conclusões relativamente à suplementação de rotina com ferro na criança saudável até aos dois anos de idade (Figura 1).

A quase totalidade dos artigos publicados actualmente estudou populações de diferentes países, maioritariamente países em desenvolvimento, não europeus, com uma realidade diferente da portuguesa. Embora não fosse o objectivo desta revisão definir a dosagem de ferro a prescrever à criança, fica patente que os diferentes estudos apresentam também diferentes concentrações de ferro. São necessários mais estudos sobre a suplementação com ferro, orientados para a criança saudável, controlados e aleatorizados, de longa duração e de elevada qualidade, preferencialmente realizados na população portuguesa.

É deste modo importante ressalvar que o facto de os estudos incluídos nesta revisão não terem sido realizados para a população portuguesa, pode condicionar a interpretação das conclusões e actuações diferentes por reflectirem uma realidade distinta.

\section{REFERÊNCIAS BIBLIOGRÁFICAS}

1. U.S. Preventive Services Task Force. Screening for iron deficiency anemia-including iron supplementation for children and pregnant women. Rockville, MD: Agency for Healthcare Research and Quality, May 2006. Disponível em: http://www.ahrq.gov/clinic/uspstf06/ironsc/ ironrs.htm [acedido a 01/10/2008].

2. Killip S, Bennett JM, Chambers MD. Iron deficiency anemia. Am Fam Physician 2007 Mar 1; 75 (5): 671-8.

3. Palaré $M J$, Ferrão $A$, Carreira $M$, Morais A. Défice de ferro na criança. Acta Pediatr Port 2004; 35: 243-7.

4. Sachdev H, Gera T, Nestel P. Effect of iron supplementation on physical growth in children: systematic review of randomised controlled trials. Public Health Nutr 2006 Oct; 9 (7): 904-20.

5. Yurdakök K, Temiz F, Yalçin SS, Gümrük F. Efficacy of daily and weekly iron suplementation on iron status in exclusively breast-fed infants. J Pediatr Hematol Oncol 2004 May; 26 (5): 284-8.

6. Stoltzfus RJ, Heidkamp R, Kenkel D, Habicht JP. Iron supplementation of young children: learning from the new evidence. Food Nutr Bull 2007 Dec; 28 (4 Suppl): S572-84. 
7. Maestre AM, Villares JM. Suplementos nutricionales. In: Sociedad de Pediatría de Madrid y Castilla-La Mancha; Comité de Nutrición de la Asociación Española de Pediatria. Manual práctico de nutrición en pediatría 2007. Madrid: Editorial Ergon; 2007. p. 221-33.

8. Baghurst K. Include lean meat, fish, poultry and/or alternatives. In: National Health and Medical Research Council. Dietary guidelines for children and adolescents in Australia. Canberra: National Health and Medical Research Council; 2003. p. 99-122.

9. Nagpal J, Sachdev HP, Singh T, Mallika V. A randomized placebo-controlled trial of iron supplementation in breastfed young infants initiated on complementary feeding: effect on haematological status. J Health Popul Nutr 2004 Jun; 22 (2): 203-11.

10. Iannotti LL, Tielsch JM, Black MM, Black RE. Iron supplementation in early childhood: health benefits and risks. Am J Clin Nutr 2006 Dec; 84 (6): 1261-76.

11. Arnon S, ShiffY, Litmanovitz I, Regev RH, Bauer S, Shainkin-Kestenbaum $R$, et al. The efficacy and safety of early supplementation of iron polymaltose complex in preterm infants. Am J Perinatol 2007 Feb; 24 (2): 95-100.

12. Ebell MH, Siwek J, Weiss BD, Woolf SH, Susman J, Ewingman B, et al. Strength of recommendation taxonomy (SORT): a patient-centered approach to grading evidence in the Medical Literature. Am Fam Physi- cian 2004 Feb 1; 69 (3): 548-56.

13. Sachdev H, Gera T, Nestel P. Effect of iron supplementation on mental and motor development in children: systematic review of randomised controlled trials. Public Health Nutr 2005 Apr; 8 (2): 117-32.

14. Ramakrishnan $U$, Nguyen $P$, Martorell R. Effects of micronutrients on growth of children under $5 \mathrm{y}$ of age: meta-analyses of single and multiple nutrient interventions. Am J Clin Nutr 2009 Jan; 89 (1): 191-203.

15. Fischer Walker CL, Baqui AH, Ahmed S, Zaman K, El Arifeen S, Begum $\mathrm{N}$, et al. Low-dose weekly supplementation of iron and/or zinc does not affect growth among Bangladeshi infants. Eur J Clin Nutr 2009 Jan; 63 (1): 87-92.

Os autores declararam não possuir conflitos de interesses.

\section{ENDEREÇO PARA CORRESPONDÊNCIA}

Luís Filipe Cavadas

Centro de Saúde da Senhora da Hora, Rua da Lagoa, s/ $n^{\circ}$.

4460 Senhora da Hora

E-mail: luisfilipemcavadas@gmail.com

Recebido em 16/12/2009

Aceite para publicação em 25/04/2010

\section{ABSTRACT}

\section{IRON SUPPLEMENTATION IN HEALTHY CHILDREN UP TO TWO YEARS OLD - EVIDENCE BASED REVIEW}

Objective: To determine whether routine iron supplementation, in healthy children up to two years old, affects the psychomotor development, the anthropometry and morbimortality.

Data sources: MEDLINE database and evidence-based medicine sites.

Review methods: The authors searched evidence-based reviews, guidelines, meta-analysis, systematic reviews and randomized controlled trials, using the MeSH terms: Iron, Infant, Dietary Supplements. The search was limited to articles published between January 2000 and December 2009, in English, French, Spanish and Portuguese. The Strength of Recommendation Taxonomy of the Journal American Family Physician was used to assess the Level of Evidence.

Results: We found a total of 163 studies, of which we selected nine. One guideline, two evidence based reviews, two meta-analysis, one systematic review and three clinical trials met the inclusion criteria. Among the studies reviewed, there was no influence on psychomotor development, anthropometry, morbimortality in healthy children without risk factors and supplemented with iron. In children with increased risk for iron deficiency anemia the iron supplementation has proved to be beneficial.

Conclusions: There is currently no evidence of benefit for routine iron supplementation in children without risk factors up to two years old. Routine supplementation with iron for up to twelve months of age is recommended in children who have an increased risk of iron deficiency anaemia (Strength of Recommendation B - SOR B). Additional controlled, randomized and high quality studies about iron supplementation in healthy children are needed.

Keywords: Iron; Infant; Dietary Supplements. 